\title{
LA COVID-19, MOTOR DE CAMBIO DE LA TRANSFORMACIÓN EDUCATIVA MÁS GRANDE DE LOS ÚLTIMOS SIGLOS
}

\author{
COVID-19, THE ENGINE OF CHANGE IN THE GREATEST EDUCATIONAL \\ TRANSFORMATION IN RECENT CENTURIES
}

\author{
COVID-19, O MOTOR DA MUDANÇA NA MAIOR TRANSFORMAÇÃO \\ EDUCACIONAL DOS SÉCULOS RECENTES
}

\author{
Antonio Rodríguez Jiménez \\ Universidad Autónoma de Guadalajara, México \\ https://orcid.org/0000-0003-4387-7649 \\ arodriguezj15@gmail.com
}

\section{Recibido: 21/05/2021 Revisado: 01/07/2021 Aceptado:15/07/2021 Publicado: 21/09/2021}

Resumen: Los cambios en época de COVID han transformado la comunicación educativa hasta límites insospechados. La tecnología se ha adueñado de la literatura educativa o discurso literario de la educación hasta desfigurarlo, trastornando la visión tradicional de los centros de enseñanza y metamorfoseando hasta el propio desenvolvimiento de los profesores y sus hábitos, de modo que unos han desaparecido, y otros se transformaron adoptando giros de 180 grados. El objetivo de este estudio consiste en demostrar cómo se vive una de las transformaciones educativas más grandes de los últimos siglos. La metodología para elaborar este trabajo posee un carácter mixto, pero mucho más cualitativo que cuantitativo y las herramientas son artículos publicados casi todos a lo largo del 2020, así como observaciones directas de la propia experiencia en el campo de la educación, que es de donde se han podido extraer datos recientes. El resto es parte de la experiencia investigadora y de los conocimientos como educador universitario. Como conclusión, se puede llegar al fin de la escuela presencial tal y como la conocemos, aunque antes se vivirá durante algún tiempo un modelo puramente híbrido, como puede deducirse de la comparación o ejemplificación de la educación de países dispares de diferentes lugares del mundo.

Palabras claves: COVID; Educación; Transformación; Desaparición; Escuela.

Abstract: The changes in the time of COVID have transformed educational communication to unsuspected limits. Technology has taken over the educational literature or literary discourse of education to the point of disfiguring it, upsetting the traditional vision of schools and metamorphosing even the development of teachers and their habits, so that, some habits have disappeared and others have been transformed by adopting 180 degree turns. The objective of this study is to demonstrate how one of the greatest educational transformations in recent centuries is experienced. The methodology to prepare this work has a mixed character, but much more qualitative than quantitative, and the tools are articles published almost all throughout 2020, as well as direct observations of one's own experience in the field of education, which is where recent data could be extracted. The rest is part of the research experience and knowledge as a university educator. Thus, the end of the classroom-based education system may be near. Before that, however, we will likely see a purely hybrid model of education, as can be deduced from the comparison and exemplification of education systems from various countries in different parts of the world.

Keywords: COVID; Education; Transformation; Disappearance; School. 
Resumo: As mudanças na época do COVID transformaram a comunicação educacional em limites insuspeitados. A tecnologia se apoderou da literatura educacional ou do discurso literário da educação a ponto de desfigurá-la, perturbando a visão tradicional das escolas e metamorfoseando até o desenvolvimento dos professores e seus hábitos, de modo que alguns desapareceram, outros desapareceram. Voltas de 180 graus. O objetivo deste estudo é demonstrar como se vive uma das maiores transformações educacionais dos últimos séculos. A metodologia de elaboração deste trabalho tem um caráter misto, mas muito mais qualitativo do que quantitativo, e as ferramentas são artigos publicados quase todos ao longo de 2020, bem como observações diretas da própria experiência na área da educação, onde os dados recentes poderiam ser extraído. $\mathrm{O}$ resto faz parte da experiência de pesquisa e do conhecimento como educador universitário. Em conclusão, o fim da escola presencial como a conhecemos pode ser alcançado, embora um modelo puramente híbrido seja vivido por algum tempo antes, como se pode deduzir da comparação ou exemplificação da educação de países díspares em diferentes partes do mundo. Palavras-chave: COVID; Educação; Transformação; Desaparecimento; Escola.

Cómo citar este artículo: Rodríguez, A. (2021).La COVID, motor de cambio de la transformación educativa más grande de los últimos siglos. .Hachetetepé. Revista científica en Educación y Comunicación, (23), 1-11. https://doi.org/10.25267/Hachetetepe.2021.i23.2203

\section{INTRODUCCIÓN}

El punto de vista de este trabajo parte de un objetivo que interrelaciona elementos como la comunicación de la literatura educativa y el componente disruptivo es la COVID, que ha cambiado la vida de las escuelas y centros de enseñanza superior hasta límites insospechados. En los niveles más básicos se han creado problemas emocionales por la distancia y por la falta de instrumentos que han provocado que el próximo curso cuente con muchos alumnos menos en los niveles de educación básica y secundaria, y en buena escala a nivel universitario. ¿Qué ha pasado con ese alumnado desaparecido? Todo ha sido fruto de la pandemia, pero sobre todo de la pérdida de puestos de trabajo de sus padres y de la falta de computadoras para seguir las clases a distancia. La adaptación tecnológica ha sido tan frustrante que los alumnos han desaparecido del panorama escolar para siempre. La metodología para elaborar esta investigación posee un carácter mixto, pero mucho más cualitativo que cuantitativo, y las herramientas son artículos publicados casi todos a lo largo del 2020, que es de donde se han podido extraer datos actualizados.

El resto es parte de la experiencia investigadora del autor de estas líneas y de los conocimientos como educador universitario. La hipótesis de este trabajo es que es posible que se mejore la enseñanza con herramientas a distancia, y la discusión gira en torno a convencer a los alumnos de la bondad de la materia que están aprendiendo y que la disfruten como si se tratara de su asignatura más importante. La atención permanente y personalizada del profesor con los alumnos es independiente de si se realiza presencial $u$ online. Provocar la entrega de unos y de otros es la comunicación del acto educativo que produce el milagro del aprendizaje y la enseñanza.

\section{ELEMENTOS BÁSICOS DE LA RUPTURA COMUNICATIVA EN LA EDUCACIÓN}

Hace muchos años, cuando el periodismo era una materia importante de la comunicación, Hybels y Weaver (1974) observan de forma tradicional la relación entre ambas. Hoy día sigue llamando la atención, aunque ha evolucionado mucho en estos casi cincuenta años, pero realmente, al producirse el retroceso y la parálisis de la COVID-19,

e-ISSN:2172-7910

Doi: 10.25267/Hachetetepe.2021.i23.2203

Universidad de Cádiz 
se advierte que aquel libro vuelve a tener actualidad con sus gratificantes elementos comunicativos, psicológicos y educativos. Por lo que sirve de base para el arranque de este pequeño estudio.

Se parte de la idea, en esta breve investigación, que, en aquellos años, e incluso, actualmente, hay millones de personas obsesionadas por la comunicación. Todo arranca de que la sociedad americana en general, y Estados Unidos y México en particular, han experimentado en estos cincuenta años grandes cambios en su estructura social. El mundo ha ido transformándose de manera que en el siglo pasado mucha gente nacía, vivía, trabajaba y moría en la misma comunidad. Cuando unos años después comenzaron los cambios constantes, el afán de una especie de nomadismo que hacía al ser humano más cambiante. Véase el ejemplo de México y de otros países del medio y sur americano cuyo afán por mejorar provocaba la emigración a Estados Unidos en busca de la quimera de vivir en una sociedad más rica que le proporcionara un bienestar social imposible de hallar en su lugar de origen. El efecto de estos cambios lo promueve el motor gigante de la comunicación, que ha ido evolucionando hasta convertirse en una máquina ciclópea de la que disponen de miles de dispositivos que cada uno tiene en su casa y disponen de ellos hasta los hijos pequeños. La comunicación se expande como un tsunami, que lo inunda todo y lo transforma para siempre.

Todos los trastornos sociales los produce la comunicación en los años sesenta, que evoluciona de tal forma que algunas décadas después el ser humano puede estar en todas partes sin moverse de casa. De ahí que la hecatombe producida en marzo de 2020 habría sido mucho más trágica si se hubiera producido cincuenta años atrás. En el año 2020, todo se estaba preparando para la caída de la enseñanza presencial, debido a la COVID, pero, de repente, todo se aceleró con la pandemia, y, en cierta medida, sorprendió al colectivo de la educación, a pesar de que se venía acomodando en algunos centros académicos. Ayudó que existían desde hacía tiempo universidades a distancia que funcionaban con normalidad y esa tecnología pudo exportarse a la mayoría de los niveles educativos.

La desconexión física de la presencia en clase fue traumática para todos: no se podía salir a la calle, no se estaba preparado para una comunicación fluida y apenas existían sistemas de enseñanza a distancia, que realmente se acrecentaron con la magnitud de la pandemia. Se rearmaron las universidades y tardaron poco tiempo en darse cuenta de que era necesario apostar fuerte por los medios digitales para un futuro inmediato, que había cambiado las estrategias ofensivas del mundo y así se construyeron respuestas útiles, que tuvieron un costo importante. Los estudiantes se negaban a la fórmula de no poder asistir al campus donde estaban sus compañeros y profesores o a la ciudad en la que se habían independizado de sus padres y podían vivir con cierta libertad. Empezaron los afectados, los contagiados, los fallecidos y el miedo se apoderó de millones de personas en todo el mundo. Ante el desafío del cambio de vida, muchos dejaron los estudios, unos, afectados por la crisis, y otros, por creer que la fórmula de enseñanza no era válida.

Los profesores y los propios alumnos tuvieron que afrontar la crisis emocional, primero, y después un esfuerzo de aprendizaje de las TIC fuera de serie. Los cursos se sucedían sobre fórmulas nuevas de aprendizaje, sobre cómo enseñar a través de las plataformas y de permitir a la vez que los alumnos fueran entes invisibles, cuyos nombres aparecían en Teams, Zoom o Meet y a los que rara vez les veíamos las caras. El único rostro visible era el del profesor. Pero el fenómeno actual supone una auténtica 
disrupción. Esta definición llega a su máximo esplendor el día en que el mundo, la educación, la economía, las humanidades, la psicología y muchas otras ciencias, deben pararse unos instantes y de ahí surge una reflexión que afortunadamente se venía preparando y los menos jóvenes la veían absurda, pero no hubo más remedio que hacer el esfuerzo y adaptarse a los nuevos tiempos. Curiosamente se produce en los estudiantes un transitorio periodo de parálisis. Ellos -los más avanzados- se quedan atónitos y empiezan a desertar. Es precisamente el profesorado, que se fortalece en apenas un par de meses, el que los convence para que sigan una vida normal de aprendizaje a través de esos medios maravillosos creados por la humanidad cuando llegara una explosión de grandes dimensiones que solo afectara a la salud de miles de personas y a su estado emocional. Es la hecatombe del cambio, que afortunadamente nunca llega a afectar a la electricidad, pues en ese caso el apagón habría supuesto otro tipo de supervivencia.

Pasado ya más de un año y dañado todo el aparato económico de algunos países, la enseñanza sigue en la distancia y los alumnos se amoldan a los nuevos sistemas, aunque ocultos en sus cavernas platónicas. Un día de marzo del 2021 se comunica al profesorado mexicano que es hora de volver. Las clases, las aulas están preparadas con cámaras robóticas (Polycom), con artilugios que sirven para que unos pocos alumnos -los justos para que no haya contagios- asistan a las clases, y los que vivan lejos o tengan miedo al contagio, puedan permanecer ante su laptop lo mismo que lo han hecho durante un año de sus vidas. Se les comunica a los alumnos, encienden las cámaras y se quedan mirando al profesor. ¿Qué os pasa?, se les pregunta. Y ellos contestan: "Profe, no estamos preparados". Han estado tan encerrados en sus torres de marfil, que han simpatizado con la enfermedad y sus restricciones, de tal modo que ahora sufren el síndrome de Estocolmo. Es decir, han congeniado con su raptor, ese COVID asesino y disruptor que ha logrado cambiar la visión del mundo. Los alumnos ya no quieren venir a clase. Se ven las aulas vacías, con profesores ensayando el día en que se produzca el milagro total de la vuelta. Algo se ha roto además de la salud: lo principios fundamentales de la comunicación tradicional.

Si antes estaba fracturada hasta el punto de pasar junto a un vecino del bloque de apartamentos y no se le decía ni buenas tardes, y sin embargo, se adoraban a seres desconocidos que se conocían en Facebook, ese fue el principio de la ruptura de la comunicación, esa misma que hizo que desaparecieran cientos de rotativos en el mundo y que las televisiones se alivianaran en programas de puro chismorreo con seres que eran famosos porque aparecían en TV. Se trataba de personajes que no habían hecho hazaña social alguna. No eran famosos deportistas, actores, directores de orquesta ni grandes médicos, eran solo fruto de la mediocridad de la mal entendida comunicación de masas.

Cuando cambia el proceso de comunicación entre las personas, todo cambia, pues es una caída de naipes de un gran castillo que ha dejado de existir y la mudez y el silencio sobresalta al mundo. Desaparecen los apretones de manos, los abrazos, los besos. El ser humano está en un mundo que apenas roza con su puño la camisa del viejo amigo al que antes abrazaba. Las amigas no se besan, pues han llegado al grado cero de la sensibilidad humana. Las sillas de nuestros escasos visitantes están a metro y medio de distancia y siempre nos lavamos las manos y desinfectamos nuestra mesa de trabajo cuando alguien se acerca y nos conmueve con su aliento de cera líquida. ¿En qué se ha convertido el ser humano? ¿Qué hace para convencer a los alumnos de que vuelvan a las aulas? ¿Cómo se pueden aportar nuevas metodologías educativas si las distancias casi lo impiden por instantes? 
La comprensión y la facilidad de la comunicación interpersonal depende de cada uno de los individuos que están involucrados en la transacción (Hybels y Weaver, 1974). Las habilidades de la comunicación han dado un giro de 180 grados y sus experiencias socioculturales son constantes de una situación a otra, y por ello, pueden ser predecibles. De toda la nueva situación comunicacional, lo que más gusta es que se ha vuelto a la escritura. La carta se llama chat de Facebook o de whatsApp. Se escribe mal, pero prevalece el mensaje escrito o grabado. Es decir, desde mucho antes de la COVID ya se estaba iniciando la disrupción, pero lo que antes era más o menos un juego o diálogo especial entre personas de diferentes estatus sociales, ahora se ha convertido en una obligación común. Sin ella, casi no ha existido en este pasado año posibilidad de entrar en la enseñanza.

\subsection{El origen de la disrupción educativa}

La COVID, aparte de hacer sufrir a la humanidad una serie de pérdidas trágicas, ha provocado la transformación de los sistemas de enseñanza. En México, más de 30 millones de estudiantes y dos millones de docentes están enfrentándose a esta situación. En este contexto, una investigación promovida por la Universidad Iberoamericana de México, titulada Encuesta Nacional a Docentes ante la COVID-19, tuvo por objeto describir las actividades que docentes mexicanos llevan a cabo para dar continuidad al año académico, a través del cuestionario END-Covid-19, que respondieron en línea 2,253 profesores de escuelas públicas. De los resultados surge una clara descripción de las herramientas digitales utilizadas por los docentes mexicanos tanto para la gestión del curso, como para la enseñanza y el aprendizaje de los alumnos, orientadas a dar continuidad a la educación interrumpida. Combinan para ello herramientas digitales con recursos tradicionales. Se encontraron diferencias significativas dadas por región geográfica y nivel educativo; no así en cuanto a edad o sexo del profesor. Otros hallazgos importantes surgen a partir de un análisis de sentimientos docentes ante la situación que viven y, sobre todo, acerca de la capacitación y el acompañamiento que les gustaría recibir (Baptista et al., 2020).

En un artículo publicado en el periódico El Día de La Plata (Buenos Aires) se observaba una especie de reflejo mexicano, de un parecido idéntico. Su autor (Altavista, 2020 , p. 25) sostenía sin ambages que el problema de la falta de ordenadores ha tomado por sorpresa tanto a familias pudientes como a otras de escasos recursos. En su brillante artículo titulado "La pandemia que desnudó en toda su dimensión la desigualdad educativa", explicaba que hay alumnos con acceso a modernas plataformas y otros que tienen un celular sin wifi para compartir con los hermanos. El periodista Altavista (2020) escribe que hay "familias que acompañan y niños que viven en soledad. Es decir, que la inequidad del sistema educativo está en carne viva". En el artículo-reportaje del citado periodista realiza una entrevista a una maestra llamada Silvia que destaca que en este proceso de escasez las autoridades se apoyan mucho en los directores y “confían" en ellos, pero la maestra comentó que la plataforma Classroom sería la ideal en esta situación, pero de los 450 estudiantes que tiene el establecimiento educativo, apenas unos 70 pueden utilizarla (15\%). Del número de alumnos existentes en ese colegio se comprobó que 250 familias no tenían conectividad. El mismo Altavista le preguntó a otra docente que si en la contingencia se notan los colegios privados de los que no lo son. Y esta respondió que "no tanto, pues estamos percibiendo mucha disparidad en cuanto al acompañamiento familiar para con los chicos". La vuelta, pues, tendrá como siempre un papel esencial por 
parte del maestro, que, sobre todo, tendrá que hacer un gran esfuerzo para ayudar a que recuperen las emociones los alumnos de los primeros cursos, que han perdido el tiempo sin ordenadores y sin profesores, por lo que se tendrá que hacer un esfuerzo especial para rescatar en este año de supremo vacío que se ha vivido en el mundo entero.

En las universidades han existido también momentos de mucha peligrosidad, pues cuántas docenas de veces nos han dicho los alumnos que no podían asistir a clase digital porque perdían la conectividad. De repente fallaban los micrófonos, se desconectaban y eran incontrolables, y el profesor tenía la obligación de creerse los problemas técnicos de sus alumnos. Quizás esa sea la causa de que se produzca una resistencia al regreso.

\section{DISCUSIÓN}

Por su parte, Correa (2020) asume con un gran optimismo en su artículo "La innovación educativa en los tiempos del Coronavirus", que la creación pedagógica se tiene que llevar a cabo en los tiempos del Coronavirus" (2020, p. 222). Por tanto, el propósito de su artículo es la creación de conciencia podrá derribar muros, tumbar mitos, creencias y lograr que se genere en los profesores la motivación para realizar el cambio educativo que necesita la generación de jóvenes que estamos formando" acerca de la importancia de los cambios educativos y pedagógicos que exige el mundo de hoy y el del futuro, teniendo en cuenta los avances tecnológicos, las diferencias en intereses, formas de aprender, habilidades de las nuevas generaciones y las consecuencias medioambientales que surgen de un mundo globalizado en el que cada vez serán más complejas las posibilidades de encuentros presenciales multitudinarios.

En este mismo sentido de sacarle provecho a la pandemia escriben Almazán et al. en su artículo "Aprender e innovar en la pandemia" (2020). Para que este optimismo fuese posible, cada alumno debería disponer de un ordenador propio, pues salvados los obstáculos de la comunicación, a veces imposibles por la invisibilidad de los alumnos, es cuestión de entregarse a ellos y de que compartan la experiencia de aprender como si todos fuésemos ciegos. Cuando no se ve teóricamente se fija el oído y a veces se producen excelentes resultados. Es necesario que las clases sean muy comunicativas, aunque es verdad que en diversas ocasiones el alumno queda en una mudez extraña. De vez en cuando se pregunta si están atentos o en donde nos habíamos quedado, tras una pequeña pausa, y contestan perfectamente. Otro elemento al que el alumno se resiste mucho es a la asesoría LINC, pero cuando la prueba por primera vez se adhiere a ella una o dos veces por mes. Eso lo engola de un protagonismo que el alumno desconocía, pues, de repente se encuentra con una especie de clase para él solo donde el avance y la comprensión se producen de forma natural.

En este sistema ciego de la clase a distancia, una de las claves comunicativas de la enseñanza es manejar un anecdotario relacionado con la materia que rompa cada 30 minutos la pesadez que puede producir el enseñante para cumplir con su programa curricular. A veces, es necesario conectar con el mundo, con la realidad, con las noticias y resaltar los valores humanos de ejemplos de docentes en los casos de estudiantes de educación. Al alumno hay que transmitirle una verdad: que su materia no existe por azar sino porque es necesaria para su desenvolvimiento el día de mañana cuando ejerza su profesión. Por eso, si se imparte Metodología de la Investigación al alumno, hay que enseñarlo a estudiar y tiene que comprender la ortodoxia de sus conocimientos, pero un buen profesor debe enseñar al estudiante en esa materia a investigar, porque la mayor parte de su vida deberá investigar y es necesario inculcarle los valores de la investigación. 
En el caso de los alumnos de Epistemología se les enseña a que aprendan a construir junto al profesor el juego epistemológico del saber y que creen un modelo de estudio de análisis de textos que hagan de la reflexión un juego que les lleve al aprendizaje de la materia.

Por su parte, Rogero-García (2020) sostiene que la suspensión de las clases provocada por la COVID-19 y la decisión de continuar con el curso a distancia han implicado que el sistema educativo haya pasado a funcionar sin la presencia de alumnado y profesorado en la escuela. En su artículo escribe que, en esas circunstancias, el sistema no puede garantizar el derecho a la educación de todo el alumnado. Su objetivo es analizar qué ideas fundamentan la ficción de que sí puede hacerlo, por qué motivos se ha construido y aceptado esta ficción, y qué efectos negativos puede tener. Por último, ofrece propuestas de política pública para mitigar estos resultados. Sobre las palabras de RogeroGarcía (2020), y partiendo de que los gobiernos provean a todos los alumnos de las herramientas necesarias, es posible en los niveles superiores no solo continuar con la enseñanza a distancia sino culminar las carreras. Otra cosa sería la enseñanza primaria y secundaria, donde el contacto de acercamiento a los profesores forma parte de la educación complementada en las casas con las familias. Ahora bien, la enseñanza universitaria presencial no es solo posible sino recomendable, por lo que la idea que han tenido universidades como la UAG de implantar un sistema híbrido no solo es posible, sino recomendable. Unos alumnos podrían asistir a clase presencial y otros lo podrían hacer a distancia con la misma normalidad y así evitar la masificación pública. Los resultados son muy parecidos y enriquecedores y si se cuenta con los medios necesarios, la educación a distancia para los alumnos que vivan en lejanas latitudes es muy saludable para ellos y para la economía de los padres. Este tipo mixto, incluso podría motivar que se matricularan más estudiantes en nivel presencial sin que se masificaran las aulas.

Una visión muy interesante sobre las carencias y retos de la educación la aportan Corral y de Juan (2021) cuando se refieren al aprendizaje cooperativo, que explican como un amplio y heterogéneo conjunto de métodos de instrucción estructurados, en los que los estudiantes trabajan juntos, en grupos o equipos, ayudándose mutuamente en tareas generalmente académicas. Este tipo de aprendizaje actualmente se encuentra en auge, debido a los múltiples beneficios y ventajas que aporta en las aulas a cualquier nivel educativo. Corral y de Juan (2021) explican que esto "permite trabajar a los alumnos en grupos, cooperando entre ellos para conseguir realizar actividades propuestas por el docente. Este, por otra parte, tiene un papel secundario en el proceso de enseñanza, dejando a los alumnos ser ellos los protagonistas. Sus funciones se limitan a guiar el aprendizaje y a orientar al alumnado"

\section{1. Resultados: ¿El fin de la escuela?}

Feito (2020), en su artículo sobre el fin de la escuela tal y como se la conoce, explica que la crisis provocada por la pandemia ha situado a la escuela en un escenario inédito.

De un día para otro, la enseñanza -cuando esto ha sido posible- ha pasado de ser presencial a serlo virtual. Se desconoce tanto el porcentaje de profesores como de alumnos que pueden estar enseñando y aprendiendo telemáticamente y a qué estrategias están recurriendo. En este escenario, las diferencias en términos de capital económico y cultural se agravan. Si no se adoptan medidas radicales, es posible que este periodo sin docencia presencial pueda pasar factura a toda una 
generación. Profesores, alumnos, padres y madres están accediendo a contenidos didácticos - del tipo de la academia Khan- que ponen de manifiesto que es posible aprender de otra manera, de un modo más autónomo. Esto puede traducirse en una redefinición creativa e innovadora del papel del profesor. (Feito, 2020, p. 124) Es posible que, a partir de la vuelta a la docencia presencial, no sea preciso pasar tantas horas en la escuela y, muy especialmente, en el aula. La universidad, igualmente, debería aprovechar la ventaja de contar con más medios y con un estudiantado más conectado a la red que el del resto de los niveles educativos, para acometer una transformación profunda de su docencia. Las palabras de Feito (2020) coinciden con lo que se enunció más arriba sobre lo positivo de las clases en línea, que ahorrarán mucho transporte y comidas fuera de casa, pero todo ello de manera voluntaria, por acuerdo entre institución y alumnado, entre profesores y autoridades académicas.

Las clases online como única solución al cierre de los centros de enseñanza a causa del confinamiento por la COVID-19 han acentuado por tanto la brecha digital. Esto tendrá graves consecuencias en el ámbito de la educación, produciéndose desigualdades académicas entre los que pertenecen a familias con mayores ingresos de aquellos cuya economía es inferior, entre los que viven en entornos urbanos y los que residen en el mundo rural. Es por tanto urgente tomar conciencia de estas desigualdades para favorecer políticas encaminadas a dotar de medios a los alumnos más desfavorecidos ante el acceso al entorno digital. Solo así la educación será "igual” para todos, tal y como reza el art. 17 de la Constitución Española (Corral y de Juan, 2021). Estas afirmaciones son interesantes, pero tienen una utilidad europea y sirven menos para Latinoamérica donde las brechas son tan grandes que difícilmente se acercan a los países del llamado Primer Mundo.

\subsection{Ideales educativos con o sin pandemia}

La campeona del mundo en la enseñanza se dice que es Finlandia y la base de esta creencia es que el país cree en la importancia de la educación y un profesor es una persona muy bien formada con una capacidad comunicativa con sus alumnos asombrosa. En Finlandia, los maestros son muy valorados. La carrera docente es prestigiosa, exigente y está reservada para los más inteligentes y esforzados. La profesión docente requiere una maestría en Educación, se requieren cinco años de estudios universitarios para convertirse en un maestro cualificado. La profesión goza de un prestigio real en la sociedad finlandesa. Esto no tiene que ver con la remuneración -que se acerca al promedio de los países de la OCDE - sino con la importancia que otorga el país a su educación y al sentimiento ampliamente extendido de que los docentes son expertos en su dominio y que ellos se consagran con todo su corazón a su tarea. A la pregunta de ¿por qué ha elegido usted esta carrera?, la respuesta que aparece más a menudo en las encuestas a las que se somete a los aspirantes a la docencia es "porque quiero a los niños". Los profesores finlandeses están ampliamente inclinados hacia la comprensión de los niños y sus necesidades, y sienten que se encuentran al servicio de la infancia más que al servicio de una materia.

En ocasiones, la primera reacción de los políticos ante unos resultados decepcionantes en las comparaciones de PISA ha sido intentar aumentar las inversiones. Sin embargo, no hay una relación directa entre las intenciones de apoyar y la propia inversión en educación, que es casi irrelevante. Personas de excelente calidad que quieran ser profesores. Además, si hay que reclutar a muchos más profesores y la masa salarial es la misma, el salario de cada uno va a ser muy bajo, y esto va a disuadir sobre el estudio 
de la carrera docente a jóvenes brillantes, como ya está pasando en algunos centros del país. Es necesario atraer a las personas más capaces, ofrecerles un buen salario y mostrarles mucho aprecio, de modo que quieran quedarse en la profesión y que muchos alumnos puedan gozar del privilegio de aprender con ellos. Sin embargo, si un país quiere aplicar los resultados de estas experiencias, es probable que tenga que introducir algunos cambios: si se acepta que lo más importante para el alumno es tener un buen profesor, los sindicatos se ven frente a un gran reto. Van a tener que anteponer el bien de los alumnos y del país a su propia tradición colectivista. Es probable que tenga que cambiar la legislación laboral. Para atraer a buenos candidatos habrá que cambiar la formación docente, lo cual quiere decir librar una batalla con los departamentos de Pedagogía para quitar lo que no es realmente útil. Los finlandeses tienen en gran estima la profesión docente, lo cual contribuye a que la formación reciba muchísimas más solicitudes de las que se puedan aceptar. La docencia se convierte así en una carrera muy selectiva. El trato personal con los alumnos, el contacto diario tanto personal como a distancia es esencial, sobre todo en los niveles de primaria y secundaria.

Corea del Sur es uno de los países en los que el Estado apoya a la enseñanza como una apuesta de futuro a su sociedad. Tras la continua evolución de su sistema educativo, tradicionalmente los coreanos han dado gran importancia a la educación, no solo como un medio para la realización personal sino también como un medio de avance y reconocimiento social. Desde la teoría tradicional confucionista, el principal objetivo de la educación consistía en cultivar la moral y la ética de las personas. Sin embargo, con la industrialización, la educación se fue centrando cada vez más en cómo utilizar ésta para alcanzar los objetivos económicos. Por eso se puede decir que la educación en Corea ha sufrido numerosas transformaciones y desarrollos que han hecho cambiar su objetivo para adaptarse a las necesidades de cada tiempo. Actualmente, el sistema educativo está apostando mucho por el valor formativo que aportan las tecnologías de la información y la comunicación o las propias TIC.

Los esfuerzos para reformar la educación con el fin de introducir las TIC en el currículo han sido una constante por parte del gobierno coreano desde los años ochenta. En Corea, el Ministerio de Educación, Ciencia y Tecnología es la máxima institución educativa encargada de desarrollar las coordenadas y directrices de las políticas educativas del país, además de establecer un marco estratégico que busque la calidad y eficacia para acceder a las TIC desde la igualdad. Un punto fuerte de la política educativa coreana ha sido la apuesta por la introducción de las TIC en la educación.

Otro ejemplo educativo es Japón, que llegó a ser potencia mundial y a ser un país con grandes cambios y avances en todos los ámbitos. Desde los comienzos del siglo XX en Japón, se logró que la mayoría de los niños asistieran a la escuela y los seis años de primaria se volvieron obligatorios. Algo que caracteriza mucho a Japón es la excelencia de sus escuelas, ya que todos los centros educativos del país tienen este nivel, y que hay muy poca diferencia en cuanto a la calidad entre los sectores rurales, urbanos y suburbanos, lo que quiere decir que la mayoría de las personas tienen acceso a una educación digna y de calidad. Afirma Ramírez (1993, pp.355-369) que "dicho sistema es descentralizado, las autoridades locales se reservan para sí la educación obligatoria (escuelas primarias y secundarias) y dejan el bachillerato y, sobre todo, la educación superior, abiertos a la participación del sector privado."

Japón ha ido reformando el currículo a lo largo de los años y esto ha funcionado para garantizar a los ciudadanos una mejor educación y enseñanza. En el currículo se 
basan las actividades de la educación y el contenido que es prácticamente lo que define la calidad de la educación, entonces es una parte sumamente importante. "Además éste puede favorecer o perjudicar los deseos de estudiar, así como el desempeño de los procesos educativos de los estudiantes, por lo que tiene un fuerte impacto en la difusión y administración de la educación.” (Masami y Murata, 2005, p. 145)

Los que ayudan en los procesos de reforma curricular y avalan estos métodos son representantes del sistema educativo, desde las autoridades gubernamentales hasta los docentes que están frente a grupo; esto ayudará a que el sistema educativo mejore, ya que se pueden ver las distintas perspectivas en la educación, no solo de los cargos más altos, si no de las personas que están al frente, que en este caso son los docentes.

Japón siempre ha tenido muy claro que si el sistema educativo posee calidad, que se tenga una enseñanza y un aprendizaje eficaz, donde los alumnos piensen, desarrollen habilidades, destrezas, conocimiento, entonces se debe buscar la manera de efectuar cambios significativos en el sistema educativo, sin importar la carga y la dificultad que esto tenga.

\section{CONCLUSIONES}

Aunque no se ha mencionado en este texto, debe prorrumpir en esta parte de cierre que la creatividad es el elemento clave de la comunicación educativa, sencillamente porque es el ser humano y su capacidad de innovación el que va a cambiar las perspectivas que se han sufrido a lo largo de un año y medio y aún no han acabado, pues a pesar de que los semáforos están en verde se pueden retornar a rojo y todos volverán de nuevo a sus casas.

La cuestión es que lo sagaz, lo hábil es sacarle beneficio a esta terrible pandemia en tiempos de crisis y buscarle sus oportunidades. El sistema educativo, pero sobre todo los propios profesores saben las debilidades y fortalezas que ha traído esta pandemia. A través de ella todo el mundo aprendió las TIC, desde el enojoso Moodle hasta el dificultoso Teams, el amable Zoom, el Classroom, el fácil Meet o la plataforma Canvas. Todo el profesorado está preparado para manejar la plataforma y enseñar a sus alumnos a entrar en los foros, a hacer sus tareas o sus exámenes. Todo está listo para volver a las clases presenciales, pero ya no atrapará desprevenido a nadie una nueva pandemia o la repetición de la misma. Una vez que se solucionen los problemas técnicos de las enseñanzas básicas, todos estarán listos para cambiar los ritmos, los modos y mejorar la educación con las nuevas tecnologías. Es decir, ha llegado el momento de aprovechar estas oportunidades que se han manejado para no perderlas jamás e ir modernizándolas en la medida de lo que se pueda.

Se ha analizado en estas líneas los cambios comunicativos de las últimas décadas y sus procesos básicos hasta los más complejos; el origen de la disrupción educativa producido por la COVID, el posible fin de la escuela presencial y el comparativismo o ejemplificación de la educación de países dispares de diferentes lugares del mundo. Este es el principio del cambio, que es necesario verlo con optimismo y extraer el jugo a sus oportunidades. La educación lo merece, existe sobrada literatura para reflexionar, y la comunicación es un compromiso de todos.

FINANCIACIÓN. Esta investigación no recibió ninguna financiación externa. 


\section{REFERENCIAS BIBLIOGRÁFICAS}

Almazán Z., Loeza A., y López V. (2020). Aprender e Innovar en la pandemia. Revista El mundo de la educación, (17), 54-58.

Altavista, C. (2020). La pandemia que desnudó en toda su dimensión la desigualdad educativa. Periódico El Día. https://bit.ly/3jIDBHI

Baptista, P., Almazán, A., Loezo, C.A., López, V.A., y Cárdenas, J.L. (2020). Encuesta Nacional a Docentes ante el COVID- 19. Retos para la educación a distancia. Revista Latinoamericana de estudios Educativos, 50(1), 41-88. https://doi.org/10.48102/rlee.2020.50.ESPECIAL.96

Corral, D. y de Juan, J, (2021). La educación al descubierto tras la pandemia del COVID19. Carencias y retos. Aularia: Revista Digital de Comunicación, 10(1), 21-28.

Correa, S. (2020). La innovación educativa en los tiempos del Coronavirus. Salutem Scientia Spiritus , 6(1), 14-26. https://bit.ly/3zMnjDd

Feito, R. (2020). Este es el fin de la escuela tal y como la conocemos. Unas reflexiones en tiempo de confinamiento. RASE Revista Sociología de la Educación, 13(2), 156-163. https://doi.org/10.7203/RASE.13.2.17130

Hybels, S. y Weaver, R. (1974). La comunicación. Logos, Consorcio Editorial.

Masami, I., y Murata, T. (2005). Plan de Estudios (Currículum). CRICED.

Ramírez, J. (1993). El sistema educativo japonés: sus características y la formación escolar de la población. Estudios de Asia y África, 28(3), 355-369. https://bit.ly/3DPz3Hj

Rogero-García, J. (2020). La ficción de educar a distancia. Revista de Sociología de la Educación-RASE, 13(2), 174-182. http://doi.org/10.7203/RASE.13.2.17126. 